\title{
A Comparison of Malignant Bone Treatments for Reuse
}

\author{
Masaki Yazawa ${ }^{1}$, Taisuke Mori ${ }^{2}$, Kazuo Kishi ${ }^{1}$ \\ ${ }^{1}$ Department of Plastic and Reconstructive Surgery, Keio University School of Medicine, Tokyo, Japan \\ ${ }^{2}$ Department of Pathology, National Cancer Center, Tokyo, Japan \\ Email: yazawa@a7.keio.jp
}

Received November 6, 2012; revised December 8, 2012; accepted December 17, 2012

\begin{abstract}
The After cancer resection including bone, recently, bone resected with cancer has been considered to be reusable. We newly examined superheated steam treatment for bone reuse and compared it to existing treatments. Forty male $\mathrm{C} 3 \mathrm{H} / \mathrm{HeN}$ mice were used to establish a model of mandible invasion by oral squamous cell carcinoma (OSCC). The mice were sacrificed to harvest the tumor invading mandible bone. The resected tumor with mandible bone was treated by one of four kinds of treatment, no treatment for the control, the Pasteur method, liquid nitrogen treatment, microwave treatment and superheated steam treatment. After each treatment, the resected bone was transplanted into a syngeneic mouse back. Eight weeks after transplantation, the mice were sacrificed and evaluated pathologically. Grafted tumors showed recurrence: $7 / 7$ in the control, $6 / 8$ in the liquid nitrogen treatment, 1/8 the microwave treatment and 2/8 the superheated steam treatment groups. No recurrence, on the other hand, was observed in the Pasteur method (0/8). The Pasteur method is a good treatment to remove malignant cells.
\end{abstract}

Keywords: Pasteur Method; Bone Reuse; Microwave; Liquid Nitrogen

\section{Introduction}

After cancer resection including bone, it is basically necessary to compensate for the defect of bone by reconstructive surgery. The bone is generally autologous bone taken from another part of the patient, but is sometimes allograft or artificial bone. In cases where the bone has a complicated shape or is very large in size, adequate recovery of quality of life is not achieved by reconstructive surgery.

As one solution to this issue, recently, bone resected with cancer has been considered to be reusable. Several treatments were reported and involved attempts to remove malignant cells from the bone, for example, by alcohol treatment, radiation, autoclave, water-bath treatment (Pasteur method), microwave treatment and liquid nitrogen treatment. Each treatment, however, has certain drawbacks as well as advantages. We newly examined superheated steam treatment for bone reuse and compared them to existing treatments.

\section{Materials and Methods}

\subsection{Animal Care}

The experimental procedure was authorized and reviewed by the Keio University Experimental Animal Center Committee (Approval \#11025 (0)). Forty C3H/HeN mice (8-week-old male, body weight 25 to $30 \mathrm{~g}$; purchased from Charles River Laboratories Japan Inc., Tokyo, Japan) were used in the current study. All surgery was performed in an animal-operating suite at the university.

\subsubsection{Cell Culture}

SCC VII cells, derived from a cell line of mouse oral squamous cell carcinoma (OSCC), were cultured in DMEM with $10 \%$ fetal bovine serum and $1 \%$ antibioticantimycotic (Gibco/Invitrogen, MI, USA) in a humidified atmosphere of $95 \%$ air and $5 \% \mathrm{CO}_{2}$ at $37^{\circ} \mathrm{C}$.

\subsubsection{Animal Surgery}

Forty male $\mathrm{C} 3 \mathrm{H} / \mathrm{HeN}$ mice weighing about 25 to $30 \mathrm{~g}$ and aged 8 weeks old were used to establish a model of mandible invasion by OSCC as described previously $[1,2]$. They were maintained under specific pathogen-free conditions throughout this experiment. For the mandible invasion model, mice were anesthetized by intraperitoneal injection of Avertin (Sigma-Aldrich, \#T4, 840-2), and $1 \times 10^{6}$ viable SCC VII cells in $150 \mu$ of PBS were injected s.c. into the right masseter region. Three weeks after injection, by which time the tumor had grown to 1 $\mathrm{cm}$ in size, the mice were sacrificed to harvest the tumor invading mandible bone. The resected tumor with mandible bone was treated by one of four kinds of treatment. No treatment was applied to the control group (C). The Pasteur method involved immersion in a hot-water bath $\left(65^{\circ} \mathrm{C}\right)$ for 25 minutes $(\mathrm{P})[3,4]$. Liquid nitrogen treatment involved immersion in liquid nitrogen for 20 min- 
utes, standing at room temperature for 15 minutes, and immersion in distilled water for 10 minutes (N) [5-10]. Microwave treatment involved heating in a microwave oven (1000 W microwave mode: Healsio AX-PX1, Sharp Corporation, Japan) for 2 minutes (M) [11-15]. Finally, superheated steam treatment involved heating in a superheated steam oven $\left(80^{\circ} \mathrm{C}\right.$ superheated steam mode: Healsio AX-PX1, Sharp Corporation, Japan) for 10 minutes (S). After each treatment, the resected bone was transplanted into a syngeneic mouse back, and the skin was closed using surgical sutures. Eight weeks after transplantation, the mice were sacrificed and evaluated pathologically.

\section{Results}

The cultured SCC VII injection into masseter muscle at $0.2 \mathrm{ml}\left(1 \times 10^{5} \mathrm{ml}\right)$ produced a tumor of $1 \mathrm{~cm}$ in diameter in 3 weeks. As shown in Figure 1, the tumor grew surrounded by mandible and the tumor weight with mandible was $0.45 \mathrm{~g}$ on average.

Grafted tumors showed recurrence: As shown in Fig-
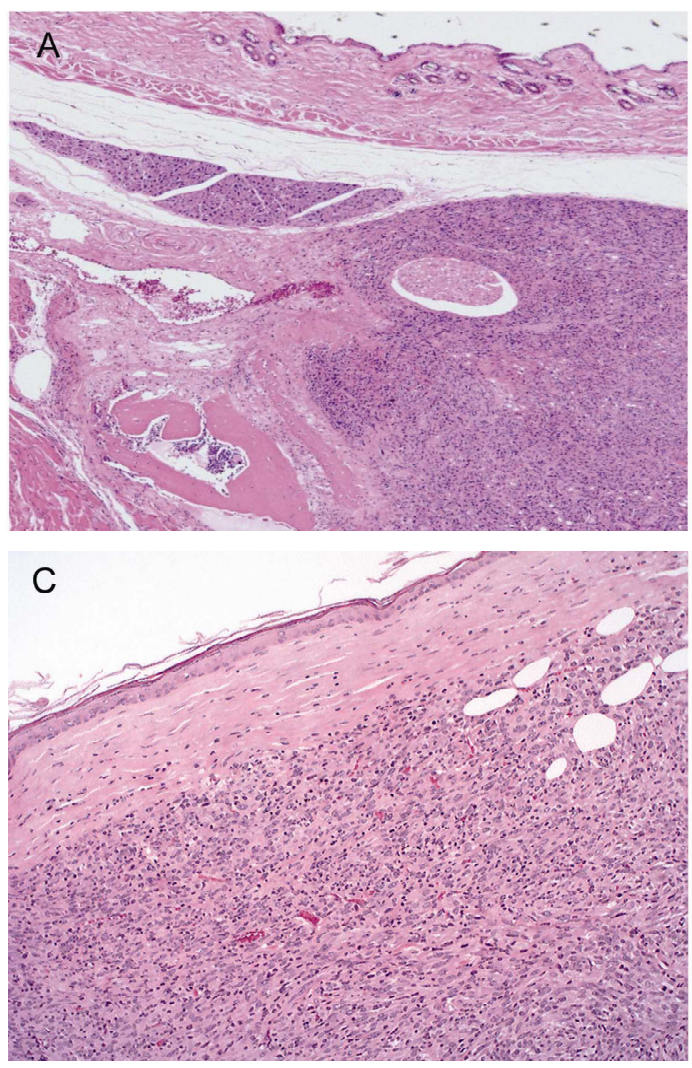

ure 2, 7/7 in the control (C), 6/8 in the liquid nitrogen treatment $(\mathrm{N}), 1 / 8$ the microwave treatment $(\mathrm{M})$ and $2 / 8$ the superheated steam treatment (S) groups. No recurrence, on the other hand, was observed in the Pasteur method (P; 0/8).

\section{Discussion}

In a cancer resection, tumor is sometimes resected with bone. The resected bone contains malignant cells, and it is difficult to remove these cells manually for reuse of the bone. From the standpoint of reconstructive surgery, there are limitations to exchanging the resected bone for autologous bone taken from another part of the patient because of its shape and size. Recently, several kinds of treatment for bone reuse have been studied, for example, alcohol treatment, radiation treatment, autoclave treatment, hot-water-bath treatment (Pasteur method), and liquid nitrogen treatment. In clinical applications, treatments for bone reuse have to remove malignant cells from bone completely and simply. Treatments that are too weak result in malignant cells remaining in bone.
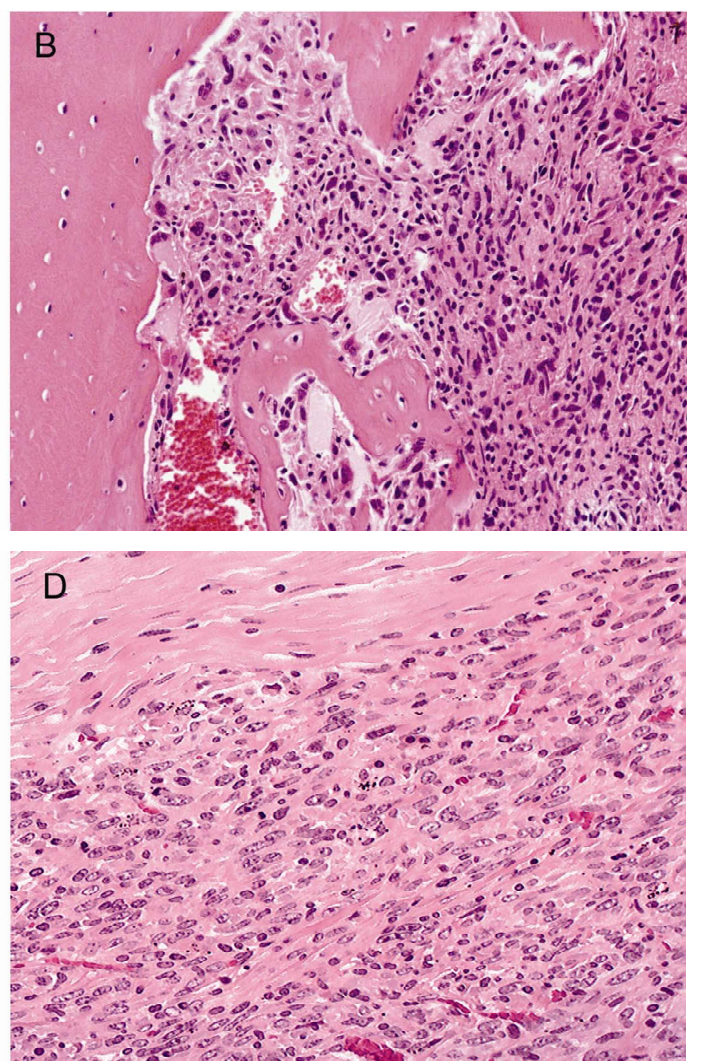

Figure 1. Animal model of maxillo-facial bone invasion and second transplantation tumor regression SCC7 cells $\left(1 \times 10^{5}\right.$ cells $)$ were implanted to establish a model of maxillo-facial bone invasion by oral squamous cell carcinoma. Microscopically, the tumor was seen adjacent to the maxilla and parotid gland (A). The tumor showed high cellularity and consisted of dyskeratinized squamous cell carcinoma. High-power view: the tumor showed marked direct bone invasion (B) H \& E. The established tumors were transplanted into the backs of syngeneic $(\mathrm{C} 3 \mathrm{H} / \mathrm{HeN})$ mice. The tumor regressed in the subcutaneous region (7/7). The tumor cells showed hyperchromasia and nuclear atypia. No degenerative change or necrosis was seen (C) and (D), H \& E. 

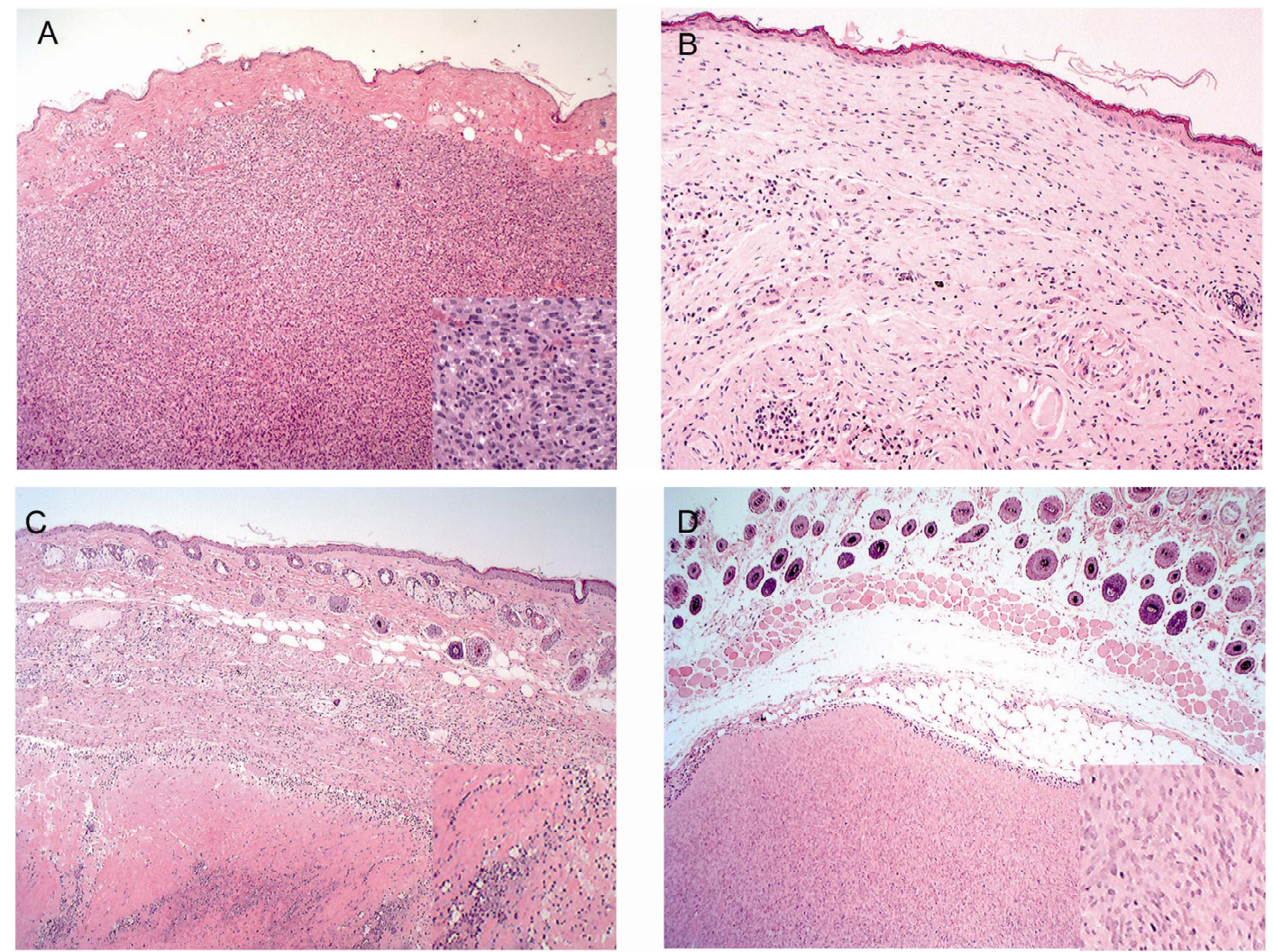

Figure 2. Pathological findings of the subcutaneous tumor-transplanted region. The liquid-nitrogen treatment group showed apparent tumor formation three weeks after transplantation (6/8). The tumors were viable and showed high cellularity without necrosis. Inset: high-power view of the viable tumor (A). No tumor was seen in the pasteurization group (0/7) (B). However, water-bath and microwave treatment groups showed low probability of tumor regression and very small ( $<3 \mathrm{~mm})$ tumor formation. Microscopically, massive necrosis and inflammatory changes were seen in the microwave treatment group (1/8) (C). High cellularity, but degenerative change and low viability of tumor, was seen in the water-bath treatment group (2/8) (D) H \& E.

Treatments that are too intense weaken bone and result in a loss of extracellular matrix, which causes a delay of bone remodeling and can trigger bone infection. In addition, some treatments require special machines, some need complicated temperature control, and others are time-consuming. As such, they are inadequate for surgery in a clinical context. In other words, existing treatments are not perfect. In this study, microwave treatment and superheated steam treatment were applied alongside existing treatments for comparison because they do not need special machines and they are equally able to treat tumors in a few minutes. In clinical applications, bone is treated without malignant soft tissue; in this study, bone with malignant soft tissue, $1 \mathrm{~cm}$ in diameter, was treated for reuse and was grafted. The results showed that, in the control group with no treatment, recurrence occurred in $100 \%(7 / 7)$ of tumors. In the liquid nitrogen group, recurrence occurred in $75 \%(6 / 8)$ of tumors, but the tumors were viable and showed high cellularity without necrosis. In the superheated steam group, recurrence occurred in $25 \%(2 / 8)$ of tumors and in the microwave group, recurrence occurred in $12.5 \%(1 / 8)$ of tumors, but tumor re- currences were smaller and developed slower than in the control group. Both treatments showed low probability of tumor regression and very small $(<3 \mathrm{~mm})$ tumor formation. Superheated steam resembles heat engineering in providing heat from the surface of a material. In the case of thick bone, the temperature difference between the surface and a certain depth is difficult to determine and depends on its thickness. On this issue, microwaves heat water inside a material, and the temperature of the material at a given depth increases sufficiently. In addition, this treatment is available using a microwave oven for home use, so it is easy and stable to use in a short period of time. In addition, materials are made sterile by treatment with microwaves. We expected that microwave treatment was the best approach for malignant bone reuse but the treatment was not enough at this time. In the Pasteur method group, there was no recurrence. This suggests the possibility that this method can eliminate all malignant cells in a mass of $1 \mathrm{~cm}$ diameter. In other words, if malignant tissue remains within bone after manual tumor removal, the Pasteur method could eliminate it within a diameter of $1 \mathrm{~cm}$. This is safer than pre- 
vious approaches. The Pasteur method is a good treatment to remove malignant cells, but it requires accurate time and temperature control. In a clinical context, it might be complicated.

\section{REFERENCES}

[1] N. Cui, T. Nomura, H. Noma, K. Yokoo, R. Takagi, S. Hashimoto, M. Okamoto, M. Sato, G. Yu, C. Guo and T. Shibahala, "Effect of YM529 on a Model of Mandibular Invasion by Oral Squamous Cell Carcinoma in Mice,” Clinical Cancer Research, Vol. 11, No. 7, 2005, pp. 27132719. doi:10.1158/1078-0432.CCR-04-1767

[2] Y. Takayama, T. Mori, T. Nomura, T. Shibahara and M. Sakamoto, "Parathyroid-Related Protein Plays a Critical Role in Bone Invasion by Oral Squamous Cell Carcinoma,” International Journal of Oncology, Vol. 36, No. 6, 2010, pp. 1387-1394.

[3] J. Manabe, A. R. Ahmed, N. Kawaguchi, S. Matsumoto and H. Kuroda, "Pasteurized Autologous Bone Graft in Surgery for Bone and Soft Tissue Sarcoma,” Clinical Orthopaedics and Related Research, Vol. 419, 2004, pp. 258-266. doi:10.1097/00003086-200402000-00042

[4] K. Sakayama, T. Kidani, T. Fujibuchi, J. Kamogawa, H. Yamamoto and T. Shibata, "Reconstruction Surgery for Patients Musculoskeletal Tumor, Using a Pasteurized Autogenous Bone Graft,” International Journal of Clinical Oncology, Vol. 9, No. 3, 2004, pp. 167-173. doi:10.1007/s10147-004-0391-7

[5] H. Nishida, H. Tsuchiya and K. Tomita, "Re-Implantation of Tumor Tissue Treated by Cryotreatment with Liquid Nitrogen Induces Anti-Tumor Activity against Murine Osteosarcoma," Journal of Bone \& Joint Surgery, Vol. 90-B, No. 9, 2008, pp. 1249-1255. doi:10.1302/0301-620X.90B9.20671

[6] H. Nishida, N. Yamamoto, Y. Tanzawa and H. Tsuchiya, "Cryoimmunology for Malignant Bone and Soft-Tissue Tumors," International Journal of Clinical Oncology, Vol. 16, No. 2, 2011, pp. 109-117. doi:10.1007/s10147-011-0218-2

[7] Y. Tanzawa, H. Tsuchiya, T. Shirai, K. Hayashi, Z. Yo and K. Tomita, "Histological Examination of Frozen Autograft Treated by Liquid Nitrogen Removed after Im- plantation,” Journal of Orthopaedic Science, Vol. 14, No. 6, 2009, pp. 761-768. doi:10.1007/s00776-009-1392-1

[8] H. Tsuchiya, S. L. Wan, K. Sakayama, N. Yamamoto, H. Nishida and K. Tomita, "Reconstruction Using an Autograft Containing Tumor Treated by Liquid Nitrogen,” Journal of Bone \& Joint Surgery, Vol. 87, No. 2, 2005, pp. 218-225. doi:10.1302/0301-620X.87B2.15325

[9] H. Tsuchiya, H. Nishida, P. Srisawat, T. Shirai, K. Hayashi, A. Takeuchi, N. Yamamoto and K. Tomita, "Pedicled Frozen Autograft Reconstruction in Malignant Bone Tumors,” Journal of Orthopaedic Science, Vol. 15, No. 3, 2010, pp. 340-349. doi:10.1007/s00776-010-1458-0

[10] N. Yamamoto, H. Tsuchiya and K. Tomita, "Effect of Liquid Nitrogen Treatment on the Proliferation of Osteosarcoma and the Biomechanical Properties of Normal Bone," Journal of Orthopaedic Science, Vol. 8, No. 3, 2003, pp. 374-380. doi:10.1007/s10776-002-0626-3

[11] R. A. Dunsmuir and G. Gallacher, "Microwave Sterilization of Femoral Head Allograft," Journal of Clinical Microbiology, Vol. 41, No. 10, 2003, pp. 4755-4757. doi:10.1128/JCM.41.10.4755-4757.2003

[12] Q. Y. Fan, B. A. Ma, X. C. Qiu, Y. L. Li, J. Ye and Y. Zhou, "Preliminary Report on Treatment of Bone Tumors with Microwave-Induced Hyperthermia,” Bioelectromagnetics, Vol. 17, No. 3, 1997, pp. 218-222. doi:10.1002/(SICI)1521-186X(1996)17:3<218::AID-BEM $\underline{7>3.0 . \mathrm{CO} ; 2-6}$

[13] M. Liebergall, C. H. Abu-Sneineh, S. Eylon, S. Mendelson, D. Segal and A. Simkin, "Effect of Microwave Oven Induced Mild Hyperthermia on Bone Viability and Strength,” Clinical Orthopaedics and Related Research, Vol. 372, 2000, pp. 272-279. doi:10.1097/00003086-200003000-00030

[14] S. S. Patel, A. A. Owida and Y. S. Morsi, "Microwave Sterilization of Bovine Pericardium for Heart Valve Applications,” Journal of Artificial Organs, Vol. 13, No. 1, 2013, pp. 24-30. doi:10.1007/s10047-010-0489-9

[15] K. Uchiyama, M. Ujihara, K. Mabuchi, N. Takahira, K. Komiya and M. Itoman, "Development of Heating Method by Microwave for Sterilization of Bone Allograft," Journal of Orthopaedic Science, Vol. 10, No. 1, 2005, pp. 77-83. doi:10.1007/s00776-004-0857-5 